\title{
Comportamento sexual de mulheres em processo de transexualização frente ao sexo
} seguro: Revisão integrativa

\author{
Sexual behavior of women in the process of transexualization in front of safe sex: Integrative review \\ Comportamiento sexual de mujeres en el proceso de transexualización frente al sexo seguro: \\ Revisión integrativa
}

Recebido: 04/01/2021 | Revisado: 05/01/2021 | Aceito: 09/01/2021 | Publicado: 11/01/2021

\author{
Sabrina Emylle Torres Fernandes \\ ORCID: https://orcid.org/0000-0002-4703-4895 \\ Universidade Federal de Pernambuco, Brasil \\ E-mail: sabrinaemylle.torres@gmail.com \\ Nemório Rodrigues Alves \\ ORCID: https://orcid.org/0000-0002-8821-872X \\ Universidade Estadual de Ciências da Saúde de Alagoas, Brasil \\ E-mail: nemodrigues@gmail.com \\ Marina Saraiva de Araújo Pessoa \\ ORCID: https://orcid.org/0000-0002-2125-5155 \\ Universidade Federal da Paraíba, Brasil \\ E-mail: marina.saraiva@live.com \\ Kélita Mirelle Oliveira Lima \\ ORCID: https://orcid.org/0000-0002-3783-2201 \\ Universidade Federal de Pernambuco, Brasil \\ E-mail: kelita_mirelle_sp@hotmail.com \\ Priscila de Oliveira Cabral Melo \\ ORCID: https://orcid.org/0000-0002-6105-2248 \\ Universidade Federal de Pernambuco, Brasil \\ E-mail: priscila.cabral@live.com \\ Tatiane Gomes Guedes \\ ORCID: https://orcid.org/0000-0001-7149-2290 \\ Universidade Federal de Pernambuco, Brasil \\ E-mail: tatigguedes@yahoo.com.br
}

\begin{abstract}
Resumo
Objetivo: Analisar as evidências científicas relacionadas ao comportamento sexual de mulheres em processo de transexualização frente ao sexo seguro. Método: Revisão integrativa da literatura, realizada nas bases de dados PUBMED, Web of Science, Lilacs, Scopus e CINAHL, para responder à questão norteadora: Quais os comportamentos sexuais de mulheres em processo de transexualização frente ao sexo seguro encontrados na literatura científica? As buscas ocorreram entre agosto e novembro de 2020, utilizando os descritores "transexualidade/ transsexualism"; "mulheres/woman"; "comportamento sexual/sexual behavior"; "sexo seguro/safe sex" e "saúde sexual/sexual health". Após a busca com os cruzamentos desses descritores, identificou-se 2.915 artigos. Ao aplicar os critérios de elegibilidade, foram selecionados dez estudos, avaliados quanto ao rigor metodológico e nível de evidência. Na extração dos dados empregou-se um instrumento validado e adaptado. Resultados: Dentre todas as estratégias de sexo seguro identificadas nos estudos, a prevenção das Infecções Sexualmente Transmissíveis - IST's por meio de ações de educação em saúde foram as mais expressivas no contexto do comportamento sexual das mulheres trans. Conclusão: Essa pesquisa possibilitou entender que as mulheres trans reconhecem a importância do sexo seguro, porém frequentemente possuem comportamentos que colocam em risco sua saúde sexual. Os fatores associados à baixa adesão do sexo seguro dessas mulheres estão relacionados a fragilidade do setor sociocultural, educacional e da saúde.
\end{abstract}

Palavras-chave: Transexualidade; Mulheres; Sexo seguro.

\begin{abstract}
Objective: To analyze the scientific evidence related to the sexual behavior of women undergoing transsexualization in the face of safe sex. Method: Integrative literature review, carried out in the PUBMED, Web of Science, Lilacs, Scopus and CINAHL databases, to answer the guiding question: What are the sexual behaviors of women undergoing transsexualization in relation to safe sex found in the scientific literature? The searches took place between August and November 2020, using the descriptors "transsexuality / transsexualism"; "Women / woman"; "Sexual behavior / sexual behavior"; "Safe sex" and "sexual health / sexual health". After searching with the intersections of these
\end{abstract}


descriptors, 2,915 articles were identified. When applying the eligibility criteria, ten studies were selected, assessed for methodological rigor and level of evidence. In extracting the data, a validated and adapted instrument was used. Results: Among all the safe sex strategies identified in the studies, the prevention of Sexually Transmitted Infections STIs through health education actions were the most significant in the context of the sexual behavior of trans women. Conclusion: This research made it possible to understand that trans women recognize the importance of safe sex, but often have behaviors that put their sexual health at risk. The factors associated with the low adherence to safe sex for these women are related to the fragility of the socio-cultural, educational and health sector.

Keywords: Transexuality; Women; Safe sex.

\section{Resumen}

Objetivo: Analizar la evidencia científica relacionada con el comportamiento sexual de mujeres en transexualización frente al sexo seguro. Método: Revisión integrativa de la literatura, realizada en las bases de datos PUBMED, Web of Science, Lilacs, Scopus y CINAHL, para dar respuesta a la pregunta orientadora: ¿Cuáles son los comportamientos sexuales de las mujeres en transexualización en relación al sexo seguro encontrados en la literatura científica? Las búsquedas se realizaron entre agosto y noviembre de 2020, utilizando los descriptores "transexualidad / transexualidad"; "Mujeres mujer"; "Comportamiento sexual / comportamiento sexual"; "Sexo seguro" y "salud sexual / salud sexual". Después de buscar con las intersecciones de estos descriptores, se identificaron 2.915 artículos. Al aplicar los criterios de elegibilidad, se seleccionaron diez estudios, evaluados según el rigor metodológico y el nivel de evidencia. Para la extracción de los datos se utilizó un instrumento validado y adaptado. Resultados: Entre todas las estrategias de sexo seguro identificadas en los estudios, la prevención de Infecciones de Transmisión Sexual - ITS a través de acciones de educación en salud fueron las más significativas en el contexto del comportamiento sexual de las mujeres trans. Conclusión: Esta investigación permitió comprender que las mujeres trans reconocen la importancia del sexo seguro, pero a menudo tienen comportamientos que ponen en riesgo su salud sexual. Los factores asociados a la baja adherencia al sexo seguro de estas mujeres están relacionados con la fragilidad del sector sociocultural, educativo y de salud.

Palabras clave: Transexualidad; Mujer; Sexo seguro.

\section{Introdução}

Gênero e sexualidade podem ser entendidos como construções socioculturais de homens e mulheres, formados por meio de práticas, sentidos e significados que, em determinado contexto, nomeiam o que pertence a um universo masculino ou feminino (Brandao \& Lopes, 2018). É possível, assim, que alguém se enxergue como mulher, sinta ser mulher e se revele socialmente como mulher, apesar de ter nascido com um corpo masculino.

Na construção da sexualidade e gênero emerge-se o processo transexualizador, que é compreendido como uma verdadeira reconstrução de gênero, uma vez que, os indivíduos transexuais se entregam à adequação corporal para apresentar determinados signos tidos como oposto ao seu sexo biológico (Ferraz \& Leite, 2016).

O processo transexualizador, incluído no contexto da sexualidade e gênero, compreende um conjunto de estratégias assistenciais implantadas no Sistema Único de Saúde (SUS). Desse modo, com o avanço no setor da saúde, o Ministério da Saúde regulamentou e ampliou medidas que favorecem o processo de transexualização, por meio das Portarias $\mathrm{n}^{\circ} 457$, de 19 de agosto de 2008 (Brasil, 2008) e no 2803, de 19 de novembro de 2013 (Brasil, 2013). O intuito de tais Portarias é ofertar o cuidado integral à população transexual, incluindo as adequações corporais de redesignação sexual por procedimentos cirúrgicos e hormonais, em conformidade com sua identidade de gênero (Santos et al., 2019).

No processo transexualizador fazem parte os indivíduos transgêneros, no qual se assemelham ao conceito de pessoas transexuais. São consideradas pessoas que biologicamente pertencem a um sexo definido, porém, psicologicamente pertencem e identificam-se a outro, se comportando segundo este. Dessa forma, os sujeitos podem revelar sua sexualidade de várias formas, podendo viver seus desejos e prazeres corporais de muitos modos (Souza \& Costa, 2016).

O processo de transexualização feminina merece destaque, principalmente por fatores que fazem com que as mesmas se tornem mais vulneráveis em seus aspectos biopsicossociais, incluindo no âmbito da sua saúde sexual. A estigmatização das mulheres transgênero reflete na discriminação, que se concretiza pela exclusão social e, como consequência, se consolida em 
várias formas de violência. Além disso, este afastamento social pode estar relacionado com a baixa escolaridade e dificuldades no mercado de trabalho, influenciando o início dessas pessoas no mercado sexual e a adoção de comportamentos arriscados, incluindo o sexo anal desprotegido com parceiros sexuais fixos, casuais ou clientes (Magno et al., 2019).

A prática do sexo experimentado por mulheres transexuais é associada muitas vezes a riscos à saúde sexual. Um fator importante atrelado ao sexo é a vulnerabilidade dessas mulheres às Infecções Sexualmente Transmissíveis (IST) pela não utilização da camisinha em suas relações sexuais. Outra questão que torna essas mulheres mais expostas a IST é a falta de uma abordagem preventiva pelos profissionais de saúde (Ferreira et al., 2018).

A transexualização feminina envolve modificações de sua identidade, repercutindo em seu corpo, onde essas mulheres assumem um novo físico e estética. As cirurgias mais realizadas em mulheres transexuais são: a rinoplastia, a cricoplastia, os implantes de silicone nos seios e nas nádegas e a construção de uma vagina utilizando o pênis do indivíduo. Dentre os cuidados pós-operatórios, destaca-se a manipulação do dilatador vaginal por até seis meses e a higiene do canal vaginal diariamente (Petry, 2015).

A cirurgia de redesignação sexual com implante da neovagina pode acarretar em várias complicações, entre elas: cavidade vaginal deficiente, vagina estreita, perfuração da neovagina, prolapso, necessidade de dilatação continua, lubrificação para o ato sexual e dispaurenia (Campos, Ferreira \& Ferreira, 2019). Isso implica dizer que o novo corpo nas mulheres transexuais pode influenciar diretamente em sua saúde sexual, incluindo a prática do sexo inseguro, por se tratar de um corpo que vai ser experimentado de uma maneira que elas desconhecem até então. Assim, essas mulheres precisam de uma abordagem mais individualizada pelos profissionais de saúde.

A saúde sexual, incluindo os aspectos que envolvem o sexo seguro, não deve se limitar a práticas centradas no diagnóstico e na prescrição. Para que a abordagem seja efetiva é necessária à inclusão de atividades preventivas integrais, repercutindo discussões abertas das vivências da sexualidade e, sobretudo, as questões de gênero nelas implicadas (Dantas \& Couto, 2018). É primordial, nesse contexto, averiguar o comportamento sexual de mulheres em processo de transexualização frente ao sexo seguro, contribuindo com o planejamento de ações que considerem as reais necessidades desse público-alvo.

A compreensão do comportamento sexual permite o acesso a escolhas no âmbito da sexualidade de maneira informada e segura, minimizando resultados de comportamentos sexuais de risco (Pimentel et al., 2016). Assim, ações destinadas, acerca do sexo seguro, a mulheres em processo de transexualização, devem ser embasadas a partir do comportamento desse público, de forma a contribuir com a saúde sexual.

A prática profissional direcionada as mulheres em processo de transexualização deve ser subsidiada através de informações e orientações acerca do sexo seguro amparadas no embasamento científico. A reunião de informações na literatura acerca da temática referida repercute beneficamente nas ações do profissional, visando uma abordagem pautada na integralidade do cuidado as mulheres incluídas no contexto da transexualização.

É necessário, pois, conhecer as produções científicas que versam sobre o comportamento sexual de mulheres em processo de transexualização frente ao sexo seguro, a fim de identificar possíveis lacunas do conhecimento voltadas à temática em questão. Destarte, este estudo objetiva analisar as evidências científicas relacionado ao comportamento sexual das mulheres em processo de transexualização frente ao sexo seguro.

\section{Metodologia}

Com vistas a responder o objetivo proposto, utilizou-se a revisão integrativa como método de pesquisa. A revisão integrativa auxilia o pesquisador na ampla análise de variadas pesquisas organizadas de forma sintetizada, contribuindo na aquisição de conhecimento baseado em evidências científicas (Okane et al., 2016). 
Essa revisão seguiu as seguintes etapas: identificação da temática; formulação da pergunta condutora; estabelecimento de critérios de inclusão e exclusão; investigação das literaturas; seleção e revisão dos estudos; avaliação crítica de cada um dos artigos e síntese dos resultados obtidos (Mendes, Silveira \& Galvão, 2008).

Para a formulação da questão de pesquisa foi utilizado o PICO (acrônimo para patient, intervention, comparison, outcomes). O uso dessa estratégia é fundamental para formular questões clínicas pautadas em palavras-chave pesquisáveis, auxiliando na prática pautada em evidências (Sousa et al., 2018).

Dessa maneira, a questão condutora constituída foi: "Quais os comportamentos sexuais de mulheres em processo de transexualização frente ao sexo seguro encontrados na literatura científica?". Assim, o primeiro elemento (P) mulheres em processo de transexualização; o segundo elemento (I) comportamento sexual; e o quarto elemento (O) sexo seguro. Nesta pesquisa não houve a utilização do terceiro elemento (C).

Os critérios de inclusão adotados consistiram em: (a) artigos originais completos publicados nos idiomas português, inglês e espanhol; (b) recorte temporal entre 2013 e 2020. Esse marco temporal justifica-se pelo fato de no ano de 2013 ter sido instituída a redefinição e ampliação da Portaria referente ao Processo Transexualizador no Brasil. Foram excluídas: revisões de literatura, cartas ao editor, anais de congressos, teses e dissertações.

Os descritores foram selecionados nos Descritores em Ciência da Saúde (DeCS) e no Medical Subject Headings (MeSH), são eles: "transexualidade/ transsexualism"; "mulheres/woman"; "comportamento sexual/sexual behavior"; "sexo seguro/safe sex" e "saúde sexual/sexual health". Tais termos foram combinados aos pares para proporcionar uma busca ampla, cujos cruzamentos em todas as bases de dados tiveram a presença do descritor principal "transexualidade/transexualism". A combinação se deu por meio do operador booleano AND, sendo esta realizada na mesma ordem de pesquisa em todas as bases de dados escolhidas (Quadro 1).

Quadro 1. Publicações identificadas nas bases de dados. Recife-PE, Brasil. 2020.

\begin{tabular}{|c|c|c|c|c|c|c|}
\hline \multirow{2}{*}{ Cruzamentos } & \multicolumn{5}{|c|}{ Bases de dados } & \multirow{2}{*}{ Total } \\
\hline & PubMed & $\begin{array}{l}\text { Web of } \\
\text { Science }\end{array}$ & Lilacs & Scopus & CINAHL & \\
\hline Transsexualism and woman & 213 & 14 & 121 & 791 & 196 & 1.335 \\
\hline Transsexualism and sexual behavior & 15 & 557 & 21 & 425 & 08 & 1.026 \\
\hline Transsexualism and safe sex & 205 & 00 & 70 & 47 & 08 & 330 \\
\hline Transsexualism and sexual health & 33 & 07 & 39 & 97 & 48 & 224 \\
\hline Total & 466 & 578 & 251 & 1.360 & 260 & 2.915 \\
\hline
\end{tabular}

Fonte: Autores.

As bases de dados eletrônicas utilizadas foram: PUBMED, Web of Science, LILACS, SCOPUS e CINAHL. E a busca aconteceu nos meses de agosto a novembro de 2020. Nessa revisão integrativa foi utilizado o fluxograma de identificação e inclusão dos estudo segundo o modelo Preferred Reporting Items for Systematic Reviews and Meta-Analyses - PRISMA (Figura 1). 
Figura 1. Fluxograma referente ao processo de seleção das dos artigos. Recife-PE, Brasil.2020.

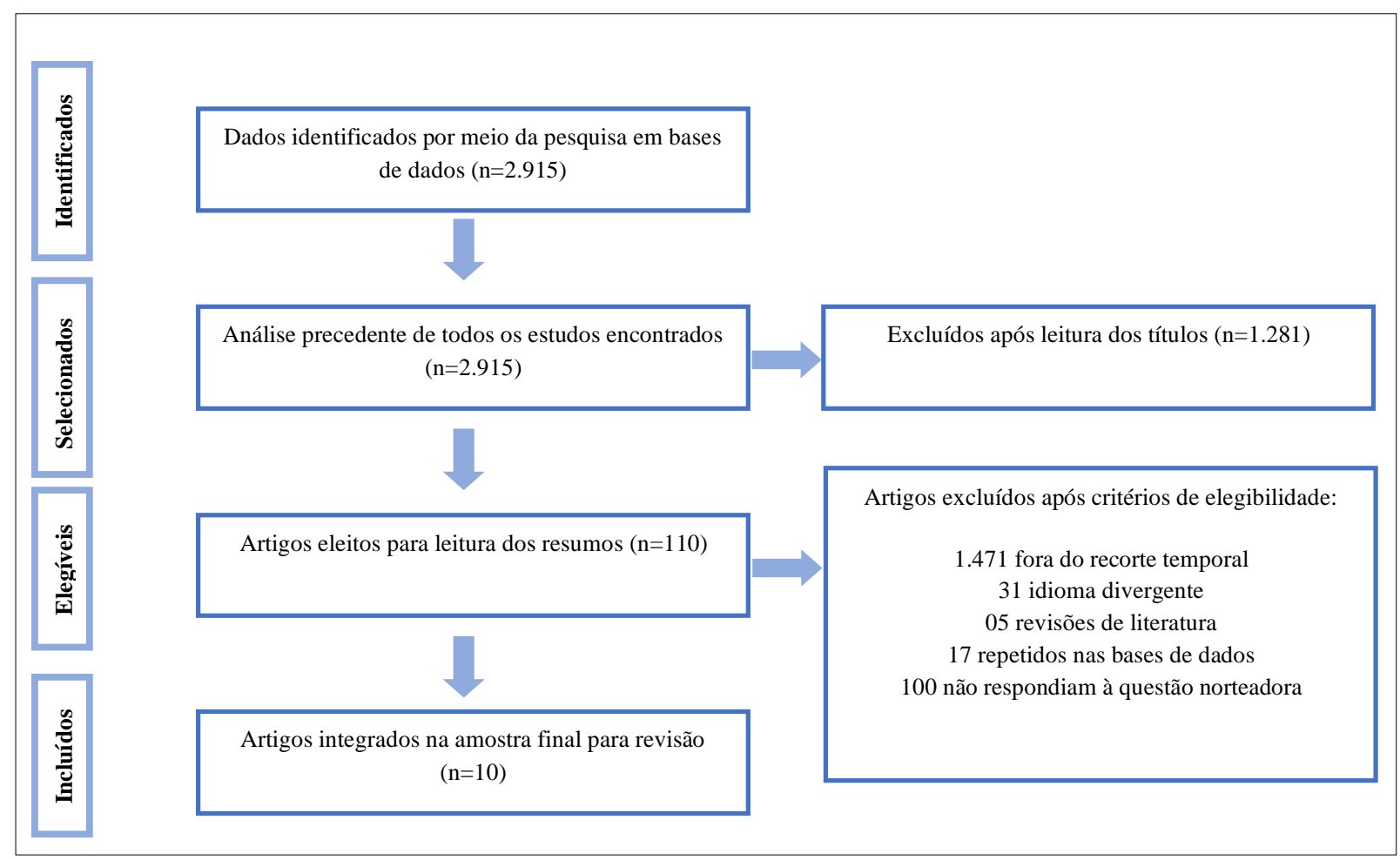

Fonte: Adaptado do modelo Preferred Reporting Items for Systematic Reviews and Meta-Analyses the PRISMA. (SHAMSEER et al., 2015)

Um formulário validado e adaptado (Ursi \& Gavão, 2006) foi preenchido com as informações dos artigos: autores, ano, país, base de dados, objetivo, nível de evidência, atitude frente ao processo de transexualização e conclusão do estudo (Quadro 2). Para avaliar o rigor metodológico a rigidez metodológica foi empregada o instrumento adaptado do Critical Appraisal Skills Programm (CASP) apropriado para cada tipo de estudo. Os artigos selecionados também foram organizados quanto a qualidade do nível de evidência, sendo esta classificada em sete níveis: o nível 1, referentes as evidências derivadas de revisão sistemática ou metanálise de ensaios clínicos randomizados; nível 2 constituídas por evidências provenientes de ensaios clínicos randomizados bem delineados; nível 3, evidências tidas como ensaios clínicos bem delineados sem randomização; nível 4, evidências compostas por estudos de coorte e de caso-controle bem delineados; nível 5, evidências originadas por revisão sistemática de estudos descritivos e qualitativos; nível 6, evidências caracterizadas de um único estudo descritivo ou qualitativo; e o nível 7, evidências provenientes de opinião de especialistas (Garcia et al., 2016).

\section{Resultados}

$\mathrm{Na}$ análise dos dez estudos selecionados, oito foram encontrados na base de dados PUBMED, seguido de um artigo no LILACS e um na Scopus. Quanto ao nível de evidência, o que predominou foi o quatro, com um total de seis artigos, seguido do cinco com três artigos e do dois com um artigo. Quanto à análise do rigor metodológico, o nível A foi avaliado em todas as publicações da amostra. Os estudos que compuseram a amostra foram publicados entre os anos de 2014-2020, sendo o ano de 2020 com mais publicações acerca da temática.

No que se refere ao idioma, prevaleceu à língua inglesa, sendo nove artigos, seguido de um artigo publicado na língua portuguesa. Os Estados Unidos da América desenvolveram grande parte das publicações relacionadas à temática. Os 
periódicos em que os manuscritos foram publicados eram, em maioria, da área médica (cinco artigos), seguidos de quatro publicações em revistas de área multiprofissional e apenas uma publicação encontrada na área da enfermagem.

Dentre todas as estratégias de sexo seguro identificadas nos estudos, a prevenção das Infecções Sexualmente Transmissíveis - IST's por meio de ações de educação em saúde foram as mais expressivas no contexto do comportamento sexual das mulheres trans. O enfoque do HIV/IST's foi revelado em quatro artigos, sendo enfatizada a estratégia de redução de risco para minimizar tais infecções, como o uso do preservativo sendo essencial. Os estudos também demonstraram implicações referentes ao comportamento sexual de risco ao HIV/IST, como ausência de preservativo nas relações sexuais, consumo de drogas e álcool, múltiplas parcerias sexuais com sexo desprotegido, baixo nível econômico e educacional (Bellhouse et al., 2016, Bhatta, 2014, Nemoto et al., 2014 \& Wilson et al., 2019).

Três artigos tiveram como foco a utilização da PrEP (profilaxia pré-exposição) como uma das estratégias de prevenção contra o HIV, abordando assim, ações prioritárias de acesso facilitado a PrEP, assim como, informações sobre seu uso, oferecimento de testes frequentes de HIV, exames de IST e promoção de preservativos (Chakprapani et al., 2020, Klein \& Golub, 2019 \& Zalazar et al., 2016).

Outra questão abordada por um dos artigos foi o estigma e preconceito ainda prevalente contra as mulheres transexuais no âmbito da saúde sexual, dificultando o acesso aos serviços de saúde a este público (Barmania \& Aljunid, 2017). A utilização de um dispositivo tecnológico em saúde como ferramenta na prevenção de HIV foi mostrada em um dos artigos, revelando que o conhecimento e atitudes sobre conexão social e acesso à saúde aumentam os comportamentos de proteção e promoção da saúde (Sun et al., 2020).

Outro resultado encontrado foi referente às representações sociais das mulheres trans que convivem com o HIV, sendo enfatizada a importância do cuidado humanizado como prioridade no atendimento a essas mulheres (Abreu et al., 2020). A síntese dos artigos encontrados está apresentada no Quadro 2.

Quadro 2. Síntese das informações dos artigos selecionados. Autores, ano, país, base, objetivo, nível de evidência, estratégia educacional e conclusão. Recife - PE, Brasil. 2020.

\begin{tabular}{|c|c|c|c|c|}
\hline $\begin{array}{c}\text { Autores/ } \\
\text { Ano/ País / } \\
\text { Base de } \\
\text { dados }\end{array}$ & Objetivo & $\begin{array}{l}\text { CASP/ Nível } \\
\text { de evidência }\end{array}$ & $\begin{array}{l}\text { Comportamento sexual } \\
\text { frente ao sexo seguro }\end{array}$ & Conclusão \\
\hline $\begin{array}{l}\text { 1. } \begin{array}{l}\text { Barmania } \\
\& \\
\text { Aljunid. }\end{array} \\
2017 \\
\text { Malásia } \\
\text { PUBMED }\end{array}$ & $\begin{array}{lr}\text { Explorar as } & \text { as } \\
\text { atitudes das partes } \\
\text { interessadas } \\
\text { envolvidas na } \\
\text { política } \\
\text { prevenção do HIV } \\
\text { na Malásia em } \\
\text { relação } \\
\text { mulheres } \\
\text { transexuais, dado } \\
\text { o contexto } \\
\text { islâmico. }\end{array}$ & Nível 5 & $\begin{array}{l}\text { Crescimento do corpo de } \\
\text { pesquisas em torno de } \\
\text { estigma e discriminação } \\
\text { contra } \\
\text { transgênero nulheres } \\
\text { em vários } \\
\text { sociedade, } \\
\text { política, incluinda } \\
\text { religiosos e } \\
\text { de saúde. }\end{array}$ & $\begin{array}{lr}\text { Atitudes } & \text { negativas } \\
\text { generalizadas, estigma e } \\
\text { a discriminação foram } \\
\text { relacionados as mulheres } \\
\text { transexuais. Isto serviu } \\
\text { para criar um ambiente } \\
\text { onde estas eram } \\
\text { consideradas } \\
\text { marginalizadas } \\
\text { portanto, um grupo } \\
\text { difícil de alcançar em } \\
\text { termos de acesso às } \\
\text { atividades de prevenção } \\
\text { do HIV. }\end{array}$ \\
\hline
\end{tabular}




\begin{tabular}{|c|c|c|c|c|}
\hline $\begin{array}{l}\text { 2. Nemoto et } \\
\quad \text { al. } \\
2014 \\
\text { Estados } \\
\text { Unidos da } \\
\text { América } \\
\text { PUBMED }\end{array}$ & $\begin{array}{l}\text { Descrever o sexo } \\
\text { anal receptivo } \\
\text { desprotegido } \\
\text { (URAS) e sexo } \\
\text { anal insertivo } \\
\text { desprotegido } \\
\text { (UIAS) entre } \\
\text { mulheres } \\
\text { transexuais em } \\
\text { relação a tipos de } \\
\text { parceiros e fatores } \\
\text { psicossociais. }\end{array}$ & Nível 4 & $\begin{array}{l}\text { Fortalecer as práticas de } \\
\text { comportamento saudável } \\
\text { e a prática do sexo } \\
\text { seguro pode reduzir o } \\
\text { URAS e UIAS com } \\
\text { parceiros das mulheres } \\
\text { transexuais. }\end{array}$ & $\begin{array}{l}\text { Este estudo forneceu } \\
\text { ressalvas que as mulheres } \\
\text { transexuais estavam } \\
\text { dispostas a se envolver } \\
\text { na UIAS com parceiros } \\
\text { comerciais, mesmo } \\
\text { sabendo que eram HIV } \\
\text { positivo. A depressão foi } \\
\text { significativamente } \\
\text { correlacionada } \\
\text { URAS com parceiros } \\
\text { principais. }\end{array}$ \\
\hline $\begin{array}{l}\text { 3. Zalazar et } \\
\text { al. } \\
2016 \\
\text { Argentina } \\
\text { PUBMED }\end{array}$ & $\begin{array}{l}\text { Avaliar a } \\
\text { disposição de usar } \\
\text { a PrEP (profilaxia } \\
\text { pré-exposição) } \\
\text { entre mulheres } \\
\text { transexuais na } \\
\text { Argentina. }\end{array}$ & Nível 4 & $\begin{array}{l}\text { Abordagem do estigma } \\
\text { baseado no gênero em } \\
\text { ambientes de saúde } \\
\text { serão fundamentais para } \\
\text { o sucesso da PrEP como } \\
\text { uma estratégia de } \\
\text { prevenção do HIV nesta } \\
\text { população. }\end{array}$ & $\begin{array}{l}\text { Foram encontrados altos } \\
\text { níveis de vontade de usar } \\
\text { a PrEP entre mulheres } \\
\text { transexuais na Argentina, } \\
\text { sugerindo que há uma } \\
\text { alta percepção de risco } \\
\text { de HIV nessa população. }\end{array}$ \\
\hline $\begin{array}{l}\text { 4. Sun et al. } \\
2020 \\
\text { Estados } \\
\text { Unidos da } \\
\text { América } \\
\text { PUBMED }\end{array}$ & $\begin{array}{lr}\text { Desenvolver } & \text { e } \\
\text { testar } & \text { a } \\
\text { usabilidade } & \text { e } \\
\text { aceitabilidade do } & \text { drans } \\
\text { protótipo Trans } \\
\text { Women } \\
\text { Connected mobile } \\
\text { aplicativo. }\end{array}$ & Nível 2 & $\begin{array}{l}\text { Intervenção de saúde } \\
\text { móvel pode apoiar } \\
\text { mudanças positivas na } \\
\text { promoção da saúde } \\
\text { sexual das mulheres } \\
\text { trans. }\end{array}$ & $\begin{array}{l}\text { Este estudo resultou em } \\
\text { um aplicativo móvel } \\
\text { aceitável que foi bem } \\
\text { recebido por mulheres } \\
\text { transgênero, sendo } \\
\text { projetado para auxiliar na } \\
\text { prevenção do HIV. }\end{array}$ \\
\hline $\begin{array}{l}\text { 5. Wilson et } \\
\text { al. } \\
2019 \\
\text { Estados } \\
\text { Unidos da } \\
\text { América } \\
\text { PUBMED }\end{array}$ & $\begin{array}{l}\text { Fornecer dados de } \\
\text { base sobre a } \\
\text { prevalência de } \\
\text { infecção à medida } \\
\text { que a cidade } \\
\text { amplia } r \text { seus } \\
\text { programas para } \\
\text { eliminar o HCV } \\
\text { (vírus da Hepatite } \\
\text { C). }\end{array}$ & Nível 4 & $\begin{array}{l}\text { Fornecimento de } \\
\text { redução de danos na } \\
\text { população trans nos } \\
\text { serviços, como troca de } \\
\text { seringas, maior acesso } \\
\text { ao tratamento de drogas } \\
\text { e educação sobre } \\
\text { transmissão podem } \\
\text { reduzir a incidência de } \\
\text { HCV entre pessoas trans } \\
\text { mulheres. }\end{array}$ & $\begin{array}{l}\text { O estudo documenta a } \\
\text { prevalência de } \\
\text { soropositividade para } \\
\text { anticorpos do HCV em } \\
\text { uma amostra de mulheres } \\
\text { trans recrutada pela } \\
\text { comunidade nove vezes } \\
\text { maior do que da } \\
\text { população em geral de } \\
\text { São Francisco. }\end{array}$ \\
\hline $\begin{array}{l}\text { 6. Bhatta } \\
2014 \\
\text { Nepal } \\
\text { PUBMED }\end{array}$ & $\begin{array}{l}\text { Examinar a } \\
\text { prevalência de } \\
\text { comportamentos } \\
\text { sexuais de risco } \\
\text { relacionados ao } \\
\text { HIV entre pessoas } \\
\text { transexuais de } \\
\text { homem para } \\
\text { mulher (MTF). }\end{array}$ & Nível 4 & $\begin{array}{l}\text { Há uma necessidade } \\
\text { urgente de programas e } \\
\text { intervenções para } \\
\text { reduzir comportamentos } \\
\text { sexuais de risco nesta } \\
\text { população minoritária. }\end{array}$ & $\begin{array}{l}\text { Idade, escolaridade, } \\
\text { renda, frequência de } \\
\text { contato sexual diário e } \\
\text { hábito de álcool } \\
\text { permanecem } \\
\text { significativo em relação } \\
\text { ao comportamento sexual } \\
\text { de risco relacionado ao } \\
\text { HIV. }\end{array}$ \\
\hline
\end{tabular}




\begin{tabular}{|c|c|c|c|c|}
\hline $\begin{array}{l}\text { 7. Chakrapa } \\
\text { ni et al. } \\
2020 \\
\text { Índia } \\
\text { PUBMED }\end{array}$ & $\begin{array}{lr}\text { Informar } & \text { os } \\
\text { programas } & \text { de } \\
\text { educação } & \text { e } \\
\text { implementação de } \\
\text { PrEP para as } \\
\text { TGW (mulheres } \\
\text { transexuais) na } \\
\text { Índia. }\end{array}$ & Nível 4 & $\begin{array}{l}\text { Treinamento } \\
\text { sensibilização de } \\
\text { profissionais de saúde } \\
\text { sobre a PrEP e as TGW, } \\
\text { questões de saúde e } \\
\text { cuidados de afirmação } \\
\text { de gênero são centrais } \\
\text { para apoiar a } \\
\text { implementação da PrEP. }\end{array}$ & $\begin{array}{l}\text { A Índia deve promover } \\
\text { informações abrangentes } \\
\text { sobre os efeitos colaterais } \\
\text { e potenciais da PrEP, seu } \\
\text { uso gratuito ou } \\
\text { subsidiado, vantagens da } \\
\text { proteção adicional no } \\
\text { trabalho sexual } \\
\text { encontros } \\
\text { forçados. } \\
\text { envolvimento } \\
\text { significativo com as } \\
\text { redes de parentesco das } \\
\text { TGW pode encorajar } \\
\text { normas da comunidade } \\
\text { transgênero sobre o uso } \\
\text { da PrEP e mitigar o } \\
\text { estigma multifacetado. }\end{array}$ \\
\hline $\begin{array}{l}\text { 8. Bellhouse } \\
\text { et al. } \\
2016 \\
\text { Austrália } \\
\text { PUBMED }\end{array}$ & $\begin{array}{l}\text { Investigar } \\
\text { demografia, } \\
\text { características, } \\
\text { comportamentos } \\
\text { de risco e } \\
\text { positividade para } \\
\text { HIV / IST entre } \\
\text { indivíduos } \\
\text { transgêneros } \\
\text { frequentando o } \\
\text { Centro de Saúde } \\
\text { Sexual de } \\
\text { Melbourne } \\
\text { (MSHC), } \\
\text { Austrália, entre } \\
\text { 2011 e 2014. }\end{array}$ & Nível 4 & $\begin{array}{l}\text { É importante considerar } \\
\text { as diferenças entre } \\
\text { indivíduos transgêneros } \\
\text { MTF (homem para } \\
\text { mulher) e FTM (mulher } \\
\text { para homem) em } \\
\text { ambientes de saúde. }\end{array}$ & $\begin{array}{l}\text { Os indivíduos } \\
\text { transgêneros neste estudo } \\
\text { foram considerados um } \\
\text { grupo diversificado, com } \\
\text { um histórico de trabalho } \\
\text { sexual sendo uma } \\
\text { característica comum. } \\
\text { Essas descobertas } \\
\text { indicam que as } \\
\text { necessidades de saúde } \\
\text { sexual dos indivíduos } \\
\text { transgêneros diferem } \\
\text { substancialmente } \\
\text { daqueles em outros } \\
\text { países, incluindo o EUA } \\
\text { e Canadá. }\end{array}$ \\
\hline $\begin{array}{l}\text { 9. Abreu et } \\
\text { al. } \\
2020 \\
\text { Brasil } \\
\text { LILACS }\end{array}$ & $\begin{array}{l}\text { Identificar as } \\
\text { representações } \\
\text { sociais } \\
\text { mulheres } \\
\text { transexuais } \\
\text { vivendo de } \\
\text { HIV/Aids. }\end{array}$ & Nível 5 & $\begin{array}{l}\text { Enaltece-se a relevância } \\
\text { dos processos } \\
\text { representacionais para o } \\
\text { cuidado em saúde de } \\
\text { forma humanizada. }\end{array}$ & $\begin{array}{l}\text { A identificação das } \\
\text { representações sociais } \\
\text { sobre o HIV/Aids } \\
\text { propiciou compreender } \\
\text { os princípios } \\
\text { organizacionais da } \\
\text { comunicação em massa, } \\
\text { que nortearam, ao longo } \\
\text { dos anos, a representação } \\
\text { das mulheres transexuais. }\end{array}$ \\
\hline
\end{tabular}




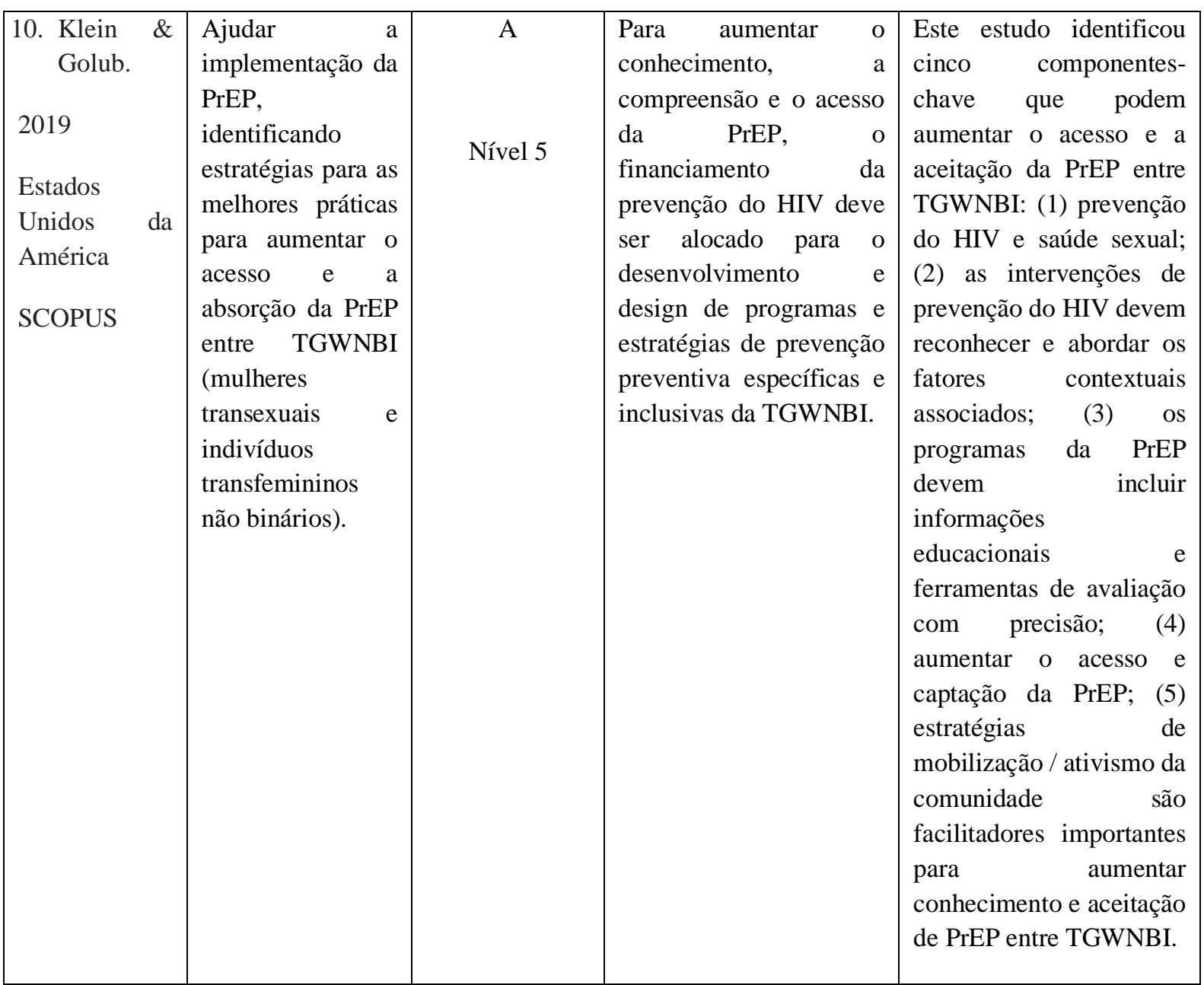

Fonte: Autores.

\section{Discussão}

O comportamento sexual de risco foi associado como empecilho ao sexo seguro das mulheres trans. O sexo anal receptivo e insertivo desprotegido entre mulheres transexuais foram evidenciados nos estudos, sendo um agravante o estado psicológico destas. A baixa autoestima, sintomas depressivos e a confiança nos parceiros sexuais foram alegações que atenuaram o sexo desprotegido (Nemoto et al., 2014 \& Bellhouse et al., 2016).

O sexo anal desprotegido constitui um elevado risco de transmissão a IST/AIDS, onde muitas mulheres transexuais se sentem forçadas pelos parceiros a não utilizarem o preservativo, o que torna fundamental medidas de empoderamento dessas mulheres na luta contra a abjeção transexual feminina e poder sobre seus corpos, saúde e a vida (Abreu et al., 2019). Ademais, programas de saúde pública devem enfatizar os riscos associados ao sexo anal desprotegido e garantir que preservativos e lubrificantes sejam acessíveis e gratuitos (Millar, 2018).

Além do sexo desprotegido, outros comportamentos sexuais de risco foram associados como negativos ao sexo seguro das mulheres trans. Dentre os estudos, evidenciou-se a prevalência da soropositividade de Hepatite C ao uso de álcool, drogas intranasais e injetáveis e o encarceramento (Wilson et al., 2019). Nesse contexto, estratégias positivas de enfrentamento e apoio social podem mitigar os efeitos negativos do uso de substancias abusivos entre mulheres transexuais. Ações que visem reduzir os fatores estressantes que atenuam o uso de drogas e álcool são essenciais, como acesso a moradia, emprego e cuidados em saúde (Hotton, 2013). 
Outro fator de risco sexual citado entre os estudos foi às mulheres trans privadas de liberdade como sendo vulneráveis a IST’s (Wilson et al., 2019). Nesse interim, o abuso sexual, a violência e a negligência de atendimento à saúde física e psicológica dessas mulheres são fatores frequentemente vivenciados por este público, sendo necessário um sistema penal que promova mudanças positivas nesses paradigmas, e como resultado, melhorias na saúde sexual das mulheres transexuais (Santos \& Gomes, 2018).

O comportamento de risco sexual relacionado ao HIV relatado entre os estudos foi associado a mulheres trans com idade de 25 anos ou mais, grande parte desempregadas, com renda de salário baixo e nível de escolaridade limitada ao ensino médio, além de multiplicidade de parceiros (mais de um parceiro ao dia) sem a utilização do preservativo e o trabalho sexual inseguro (Bhatta, 2014). Nesse sentido, as relações sociais, a falta de apoio emocional, baixo acesso a escolarização e empregabilidade são vistos como fragilidades na população de mulheres trans, sendo primordial o investimento socioeconômico e político para minimizar esses riscos apontados (Silva et al., 2020).

Como avanço a ser apontado no comportamento sexual de mulheres trans frente ao sexo seguro, a PreP foi vista como bem aceita nessa população, o envolvimento ativo da estratégia da PreP possibilita a aplicabilidade da redução de danos no que tange a remissão do HIV/AIDS, sendo repercutido nos estudos a importância do seu amplo acesso e informação (Chakrapani et al., 2020, Klein \& Golub, 2019 \& Zalazar et al., 2016). Dessa forma, a conscientização sobre a PrEP e seu alcance para entretenimento locais onde essas mulheres frequentam é de grande relevância, facilitando a divulgação sobre sua importância e seu uso (Yan et al., 2019).

O comportamento sexual das mulheres trans frente ao sexo seguro é considerado deficiente quando relacionado ao preconceito ainda existente, impossibilitando de vivenciarem o sexo seguro de maneira livre. Ao procurarem os serviços de saúde para atenderem suas demandas de saúde sexual, verbalizam que são vítimas de maus tratos e associadas a atividades ilegais, como trabalho sexual, o que constitui uma barreira significativa para a promoção e prevenção à saúde delas (Barmania \& Aljunid, 2017). O acesso aos cuidados de saúde está atrelado à disponibilidade dos serviços, sendo fundamental o enfoque na alta resolutividade das necessidades de saúde das mulheres trans (Monteiro \& Brigeiro, 2019).

As questões de religiosidade, estigma e preconceito pelos profissionais de saúde foram mencionados como pontos negativos para propiciar a efetivação de medidas preventivas no comportamento atrelado a saúde sexual das mulheres trans, incluindo a deficiência de acesso e utilização de preservativos (Barmania \& Aljunid, 2017). O preconceito da sociedade é potencializado às mulheres transexuais, envolvendo desrespeito, tabus sociais e exclusão, tais aspectos necessitam de discussões para que novas perspectivas sejam reformuladas com base na integralidade do cuidado a essa população (Silva et al., 2020).

No que tange como melhoria no aspecto do sexo seguro das mulheres trans na prevenção do HIV/AIDS, foi visto a utilização das tecnologias em saúde como um aliado na promoção da saúde sexual por meio de um aplicativo que emite uma abordagem mais específica das reais necessidades dessa população (Sun et al., 2020). Nesse contexto, o aumento acelerado do uso do telefone móvel e sua acessibilidade pelas mulheres transexuais é uma importante ferramenta de facilidade ao atendimento no âmbito da saúde sexual entre esta população (Reback et al., 2019). Assim, o envolvimento de setor educacional e tecnológico é fundamental no cuidado das mulheres trans, evidenciando estratégias de promoção e prevenção no âmbito da saúde sexual.

Para contribuir com o sexo seguro das mulheres em processo de transexualização, foi evidenciada a necessidade de se repensar nas representações sociais desse público, uma vez que os processos representacionais oferecem um amparado para discussões das necessidades específicas dessas mulheres. Foi observado que a discriminação, violência, falta de recursos, exclusão social e rejeição familiar são fatores que as distanciam de procurar os serviços de saúde diante de um diagnóstico de HIV/AIDS positivo, inviabilizando-as de ter um cuidado absoluto à sua saúde sexual (Abreu et al., 2020). 
Assim, a organização dos serviços de saúde, a formação dos profissionais de saúde, assim como o tipo de abordagem preventiva utilizada nos atendimentos é essencial para ampliar o acesso e promover o cuidado em saúde no âmbito da sexualidade (Zucchi et al., 2018). Nesse sentido, a necessidade de a equipe em saúde efetivar um atendimento humanizado, baseado no estudo das representações sociais desse público, deve ser evidenciada, abordando a individualidade de cada mulher, assim como, enfatizando a saúde sexual na sua perspectiva integral.

\section{Conclusão}

O comportamento sexual das mulheres em processo de transexualização, no contexto do sexo seguro, revelou lacunas e avanços. Nesse interim, esse estudo possibilitou entender que as mulheres trans reconhecem a importância do sexo seguro, porém frequentemente possuem comportamentos que colocam em risco sua saúde sexual. Os fatores associados à baixa adesão do sexo seguro dessas mulheres estão relacionados a fragilidade do setor sociocultural, educacional e da saúde.

As evidências científicas reunidas nesse estudo mostraram que apesar das mulheres trans emitirem comportamentos sexuais de risco, nota-se que as tecnologias em saúde e educação, a efetivação de estratégias de redução de risco, acesso à informação e aos serviços de saúde são aliados na prática do sexo seguro.

Enfatiza-se a necessidade da concretização das políticas públicas já existentes para as mulheres trans, sendo primordial que a educação em saúde, o respeito e a humanização esteja presente na realidade nos serviços de saúde que prestam assistência as mulheres em processo de transexualização. Enfatiza-se, ainda, a importância da prática de empatia as mulheres trans nos serviços de saúde, enxergando-as na multidimensionalidade do cuidado, reconhecendo-as na sua integralidade, abordando-as sempre como um ser biopsicossocial.

Por fim, evidenciou-se poucos estudos no Brasil acerca do tema em questão, assim como, a sua maioria foram publicados em periódicos médicos. Sendo assim, a produção de pesquisas na região brasileira e em revistas não só da área médica é fundamental para novos debates, envolvendo como foco questões de gênero, sexualidade e saúde sexual para as mulheres inseridas no processo de transexualização.

Sugere-se novas produções científicas acerca da temática em questão, proporcionando assim, reflexões críticas atualizadas que enfoquem o comportamento sexual de mulheres trans no contexto do sexo seguro.

\section{Referências}

Abreu, P. D., Araújo, E. C., Vasconcelos, E. M. R., Moura, J. W. S., Sousa, J. C., \& Santos, C. B. (2019). "Mulheridade” tran sexual e a emergência pelo transfeminismo: retórica do hiv/aids à luz da teoria queer. Texto \& Contexto - Enfermagem, 28, e20180294. https://doi.org/10.1590/1980-265x-tce-2018-0294

Abreu, P. D, Araújo, E. C., Vasconcelos, E. M. R., Ramos, V. P., Moura, J. W. S., Santos, Z. C., \& Santos, C. B. (2020). Social representations of transsexual women living with HIV/AIDS. Revista Brasileira de Enfermagem, 73(3), e20180390. https://doi.org/10.1590/0034-7167-2018-0390

Barmania, S., \& Aljunid, S. M. (2017). Transgender women in Malaysia, in the context of HIV and Islam: a qualitative study of stakeholders' perceptions. BMC International Health and Human Rights, 17(30), 1-10. https://doi.org/10.1186/s12914-017-0138-y

Bellhouse, C., Walker, S., Fairley, C. K., Vodstrcil, L. A., Bradshaw, C. S., Chen, M. Y., \& Chow, E. P. (2018). Patterns of sexual behaviour and sexual healthcare needs among transgender individuals in Melbourne, Australia, 2011-2014. Sexually transmitted infections, 94(3), 212-215. http://dx.doi.org/10.1136/sextrans-2016-052710

Bhatta, D. N. (2014). HIV-related sexual risk behaviors among male-to-female transgender people in Nepal. International Journal of Infectious Diseases, 22, e11-e15. https://doi.org/10.1016/j.ijid.2014.01.002

Brandao, E. R, \& Lopes, R. F. F (2018). "Não é competência do professor ser sexólogo" O debate público sobre gênero e sexualidade no Plano Nacional de Educação. Civitas-Rev. Ciênc. Soc., 18(1), 100-123. https://doi.org/10.15448/1984-7289.2018.1.28265

Brasil (2008). Portaria $n^{\circ}$ 457, de 19 de agosto de 2008. Diretrizes Nacionais para o Processo Transexualizador no Sistema Único de Saúde (SUS). Ministério da Saúde. Brasília http://bvsms.saude.gov.br/bvs/saudelegis/sas/2008/prt0457_19_08_2008.html

Brasil (2013). Portaria $n^{\circ} 2.803$, de 19 de novembro de 2013. Redefine e amplia o Processo Transexualizador no Sistema Único de Saúde (SUS). Ministério da Saúde. Brasília. http://bvsms.saude.gov.br/bvs/saudelegis/gm/2013/prt2803_19_11_2013.html 
Campos, S. R, Ferreira, M. C. S \& Ferreira, A. P. M. (2019). Repercussões Da Redesignação sexual masculino para feminino e a atuação da fisioterapia. EScientia, $11(2), 8-16$.

Chakrapani, V., Shunmugam, M., Rawat, S., Baruah, D., Nelson, R., \& Newman, P. A. (2020). Acceptability of HIV Pre-Exposure Prophylaxis Among Transgender Women in India: A Qualitative Investigation. AIDS Patient Care and STDs, 34(2), 92-98. https://doi.org/10.1089/apc.2019.0237.

Dantas, S.M.V, \& Couto, M.T. (2018). Sexualidade e reprodução na Política Nacional de Saúde do Homem: reflexões a partir da perspectiva de gênero. Sexualidad, Salud y Sociedad, 30, 99-118. https://dx.doi.org/10.1590/1984-6487.sess.2018.30.05.a

Ferraz, C.V, \& Leite, G.S (2016). A Desconstrução da Relevância Jurdica do Sexo Biológico em Face da Identidade de Gênero na Transexualidade: A Tutela Juídica da Mulher Transgênero. Conpedi Law Review, 1 (1), 67-87. http://dx.doi.org/10.26668/2448-3931_conpedilawreview/2015.v1i1.3347

Garcia, A. K. A., Fonseca, L. F., Aroni, P., \& Galvão, C. M. (2016). Estrategias para el alivio de la sed: revision integrativa de la literatura. Revista Brasileira de Enfermagem, 69(6), 1215-1222. https://doi.org/10.1590/0034-7167-2016-0317.

Hotton, A. L., Garofalo, R., Kuhns, L. M., \& Johnson, A. K. (2013). Substance use as a mediator of the relationship between life stress and sexual risk among young transgender women. AIDS Education and Prevention, 25(1), 62-71. https://doi.org/10.1521/aeap.2013.25.1.62

Klein, A., \& Golub, S. A. (2019). Increasing access to pre-exposure prophylaxis among transgender women and transfeminine nonbinary individuals. AIDS patient care and STDs, 33(6), 262-269. https://doi.org/10.1089/apc.2019.0049

Magno, L., Silva, L. A. V. D., Veras, M. A., Pereira-Santos, M., \& Dourado, I. (2019). Estigma e discriminação relacionados à identidade de gênero e à vulnerabilidade ao HIV/aids entre mulheres transgênero: revisão sistemática. Cadernos de Saúde Pública, 35, e00112718. https://doi.org/10.1590/0102$311 \times 00112718$

Mendes, K. D. S., Silveira, R. C. D. C. P., \& Galvão, C. M. (2008). Revisão integrativa: método de pesquisa para a incorporação de evidências na saúde e na enfermagem. Texto \& contexto- Enfermagem, 17(4), 758-764. https://dx.doi.org/10.1590/S0104-07072008000400018

Millar, B. M., English, D., Moody, R. L., Rendina, H. J., Cain, D., Antebi-Gruszka, N., \& Parsons, J. T. (2018). Day-Level Associations Between Substance Use and HIV Risk Behavior Among a Diverse Sample of Transgender Women. Transgender Health, 3(1), 210-219. https://doi.org/10.1089/trgh.2018.0032

Monteiro, S., \& Brigeiro, M. (2019). Experiências de acesso de mulheres trans/travestis aos serviços de saúde: avanços, limites e tensões. Cadernos de Saúde Pública, 35, e00111318. https://doi.org/10.1590/0102-311X00111318

Nemoto, T., Bödeker, B., Iwamoto, M., \& Sakata, M. (2014). Practices of receptive and insertive anal sex among transgender women in relation to partner types, sociocultural factors, and background variables. AIDS care, 26(4), 434-440. https://doi.org/10.1080/09540121.2013.841832

Okane, E. S. H., Oliveira, B. B. A., Sartori, L. P., \& da Costa Junior, V. L. (2016). Estratégias de ensino para educação e saúde: Revisão integrativa. Extensio: Revista Eletrônica de Extensão, 13(21), 138-151. http://dx.doi.org/10.5007/1807-0221.2016v12n21p138

Petry, A. R. (2015). Mulheres transexuais e o Processo Transexualizador: experiências de sujeição, padecimento e prazer na adequação do corpo. Revista Gaúcha de Enfermagem, 36(2), 70-75. http://dx.doi.org/10.1590/1983-1447.2015.02.50158

Pimentel, M. H., Preto, L. S. R., Alves, M. J. G., \& Monteiro, A. M. P. (2016). Comportamento sexual e estudantes do ensino superior. Psicologia, Saúde \& Doenças, 17(3), 352-367. http://dx.doi.org/10.15309/16psd170304

Reback, C. J., Fletcher, J. B., Fehrenbacher, A. E., \& Kisler, K. (2019). Text Messaging to Improve Linkage, Retention, and Health Outcomes Among HIVPositive Young Transgender Women: Protocol for a Randomized Controlled Trial (Text Me, Girl!). JMIR research protocols, 8(7), e12837. https://doi.org/10.2196/12837

Santos, I. P. R., \& Gomes, C. D. M. (2018). 13. Travestis no sistema carcerário do Distrito Federal: gênero e cárcere entre narrativas e normas. Revista Brasileira de Ciências Criminais, 2018, 08-31.

Santos, M. A., de Souza, R. S., da Silva Lara, L. A., de Oliveira, W. A., Alexandre, V., \& de Oliveira-Cardoso, É. A. (2019). Transexualidade, ordem médica e política de saúde: controle normativo do processo transexualizador no Brasil. Estudos Interdisciplinares em Psicologia, 10(1), 03-19. https://doi.org/10.5433/2236-6407.2019v10n1p03

Shamseer, L., Moher, D., Clarke, M., Ghersi, D., Liberati, A., Petticrew, M., ... \& Stewart, L. A. (2015). Preferred reporting items for systematic review and meta-analysis protocols (PRISMA-P) 2015: elaboration and explanation. Bmj, 349, 1-25. https://doi.org/10.1136/bmj.g7647 ps://doi.org/10.1136/bmj.g7647

Silva, R. G. D., Abreu, P. D. D., Araújo, E. C. D., Santana, A. D. D. S., Sousa, J. D. C., Lyra, J., \& Santos, C. B. D. (2020). Vulnerability in the health of young transgender women living with HIV/AIDS. Revista Brasileira de Enfermagem, 73(5):e20190046. http://dx.doi.org/10.1590/0034-7167-2019-0046

Sousa, L. M. M., Firmino, C. F., Marques-Vieira, C. M. A., Severino, S. S. P., \& Pestana, H. C. F. C. (2018). Revisões da literatura científica: tipos, métodos e aplicações em enfermagem. Revista Portuguesa de Enfermagem de Reabilitação, 1(1), 45-54.

Sousa, L. M. M. S., Marques, J. M., Firmino, C. F., Frade, F., Valentim, O. S., \& Antunes, A. V. (2018). Modelos de formulação da questão de investigação na prática baseada na Evidência. Revista investigação em enfermagem, 31-39.

Souza, M. B. D., \& Costa, J. P. R. (2016). Mulheres transexuais: quando se tornar mulher é um desafio. Seminário Nacional Demandas Sociais e Políticas Públicas na Sociedade Contemporânea.

Sun, C. J., Anderson, K. M., Kuhn, T., Mayer, L., \& Klein, C. H. (2020). A Sexual Health Promotion App for Transgender Women (Trans Women Connected): Development and Usability Study. JMIR mHealth and uHealth, 8(5), e15888. https: //doi.org 10.2196/15888 
Research, Society and Development, v. 10, n. 1, e25010111679, 2021 (CC BY 4.0) | ISSN 2525-3409 | DOI: http://dx.doi.org/10.33448/rsd-v10i1.11679

Ursi, E. S., \& Gavão, C. M. (2006). Prevenção de lesões de pele no perioperatório: revisão integrativa da literatura. Revista Latino-Americana de Enfermagem, 14(1), 124-131. https://dx.doi.org/10.1590/S0104-11692006000100017

Wilson, E. C., Turner, C., Lin, J., McFarland, W., Burk, K., \& Raymond, H. F. (2019). Hepatitis C seroprevalence and engagement in related care and treatment among trans women. J Viral Hepat, 26(7), 923-925. https://doi.org/10.1111/jvh.13089

Yan, L., Yan, Z., Wilson, E., Arayasirikul, S., Lin, J., Yan, H., \& McFarland, W. (2020). Awareness and Willingness to use HIV Pre-exposure Prophylaxis (PrEP) Among Trans Women in China: A Community-Based Survey. AIDS and Behavior, 1-9. https://doi.org/10.1007/s10461-020-03050-6

Zalazar, V., Arístegui, I., Kerr, T., Marshall, B. D., Romero, M., Sued, O., \& Socías, M. E. (2016). High willingness to use HIV pre-exposure prophylaxis among transgender women in Argentina. Transgender Health, 1(1), 266-273. https://doi.org/10.1089/trgh.2016.0033

Zucchi, E. M., Grangeiro, A., Ferraz, D., Pinheiro, T. F., Alencar, T., Ferguson, L., \& Munhoz, R. (2018). Da evidência à ação: desafios do Sistema Único de Saúde para ofertar a profilaxia pré-exposição sexual (PrEP) ao HIV às pessoas em maior vulnerabilidade. Cadernos de Saúde Pública, 34 , e00206617. https://doi.org/10.1590/0102-311X00206617 